CERN-TH/99-155

\title{
QCD running coupling effects for the non-singlet structure function at small $x$
}

\author{
B.I. Ermolaev* \\ CERN 1211, Geneve 23, Switzerland \\ M. Greco \\ Dipartimento di Fisica and INFN, University of Rome III, Rome, Italy \\ and \\ S.I. Troyan \\ St.Petersburg Institute of Nuclear Physics, 188350 St.Petersburg-Gatchina, Russia
}

\begin{abstract}
A generalization of the leading-order DGLAP evolution at small $x$ is performed for the non-singlet structure function by resumming the leading-order DGLAP anomalous dimension to all orders in the QCD coupling. Explicit expressions are obtained for the non-singlet structure function of the deep inelastic scattering, taking into account both the double-logarithmic and the single-logarithmic contributions, including the running $\alpha_{s}$ effects. It is shown that when these contributions are included, the asymptotic small- $x$ behaviour is power-like, with an exponent of about 0.4.
\end{abstract}

*Permanent address: A.F. Ioffe Physico-Technical Institute, 194021 St.Petersburg, Russia 


\section{INTRODUCTION}

Non-singlet structure functions, i.e. flavour-dependent contributions to the deep inelastic structure functions, have been the object of intensive theoretical investigation. Besides being interesting in themselves, they are also comparatively technically simple for analysis, and can be regarded as a starting ground for a theoretical description of DIS structure functions. The standard and the most widespread way for theoretical investigation of DIS structure functions is the DGLAP evolution equations [1]. As is well known, in the description of DIS, these equations provide also good agreement with experimental data [2] when they are used in the small- $x$ region, although they are not expected to work very well in this kinematical region. Actually, in the small-x region, a Regge-type behaviour for the DIS structure functions cannot be obtained from DGLAP, which predicts instead a dependence

of the type $f_{N S}=e^{\sqrt{\ln (1 / x) \ln \left(Q^{2} / \mu^{2}\right)}}$. In perturbative QCD, such Regge-like behaviour was obtained [3] a long time ago for structure functions of unpolarized DIS and recently [4,5] for structure functions of the polarized DIS. However, all those results are obtained in the leading logarithmic approximation, which has the unsatisfactory feature that $\alpha_{s}$ is regarded as a constant.

In the present paper in addition to providing formulae for the asymptotics of the nonsinglet structure function at very small $x$, we present also an explicit expression for $f_{N S}$ for small but finite values of $x$, which accounts for both leading (double-logarithmic) and subleading (single-logarithmic) contributions to all orders in QCD coupling, including running $\alpha_{s}$ effects. As logarithms of $x$ and of $Q^{2}$ are important at small $x$, we account for them both. In order to take running $\alpha_{s}$ effects into account, we use in the present work the approach of [8], with some important changes. Earlier, the running $\alpha_{s}$ effects were incorporated into the double-logarithmic equations for the non-singlet structure function at small $x$ in [4,6], but other single logarithmic contributions were not taken into account in those works. In the present work we take them into account, which makes our approach self-consistent.

In the DGLAP equations, the expressions for the anomalous dimensions take into ac- 
count a finite number of NLO contributions; in this approach, the QCD coupling depends on $Q^{2}$. After the total resummation of the singular NLO contributions to the anomalous dimensions with the double logarithmic accuracy performed in [4,5], there appeared works on the small- $x$ behaviour of the structure functions combining, to a certain extent, the results of the DGLAP with the results of [4, 5]. In particular, the works [10 13] treat the running QCD coupling at small $x$, allowing $\alpha_{s}$ to depend on the incoming photon virtuality $Q^{2}$. We arrive at a different conclusion.

The paper is organized as follows: In Sect. 2 we derive the small- $x$ evolution equations for $f_{N S}$ and solve them. In Sect. 3 we obtain the asymptotic behaviour of $f_{N S}$ and discuss it. Finally, Sect. 4 contains our conclusions.

\section{SMALL- $X$ EVOLUTION EQUATION FOR NON-SINGLET STRUCTURE FUNCTIONS}

In the Born approximation, and suppressing unimportant factors, we define the nonsinglet structure function as

$$
f_{N S}^{B o r n}=\delta(1-x)
$$

For the radiative corrections to the Born approximation in the kinematical region of small $x$ and large $Q^{2}$, both logarithms of $Q^{2}$ and of $x$ are essential and should be taken into account. Therefore, a two-dimensional evolution equation is needed for $f_{N S}$, combining the evolution with respect to both $x$ and $Q^{2}$. It is simpler to obtain such an equation

for the scattering amplitude $M\left(x, Q^{2}\right)$, with the $s$-channel discontinuity (imaginary part) proportional to $f_{N S}$ :

$$
f_{N S}=\frac{1}{\pi} \Im_{s} M\left(x, Q^{2}\right)
$$

where $s=(p+q)^{2}=p^{2}+2 p q(1-x) \approx 2 p q$ is the Mandelstam variable corresponding to the squared total energy of the process under discussion. In the Born approximation, $M$ is 
represented by the two graphs in Fig. 国, although only graph (a) has a non-zero imaginary part in $s$ and therefore only that graph contributes to $f_{N S}$. This is the reason why there is no difference in the Born approximation between the non-singlet contributions to the spin- dependent and to the spin-independent structure functions. Graphs contributing to $M$ at higher orders in $\alpha_{s}$ are obtained from both graphs in Fig.17, by adding to them gluon propagators and quark and gluon loops. Starting from the order $\alpha_{s}^{2}$, the graphs obtained from Fig.1]b, with non-ladder gluon propagators incorporated, contribute in different ways to the non-singlet components of the spin-dependent and the spin-independent structure functions (see [5]). In the present paper we consider the non-singlet contribution to the spin-independet structure functions $F_{1}$ and $F_{2}$. In terms of the Regge theory we consider the positive signature contribution to $f_{N S}$. The first-loop correction to the Born graph (a) in Fig.1 is represented by graphs in Fig.2. Having chosen the planar gauge

$$
d_{\mu \nu}=g_{\mu \nu}+\frac{k_{\mu} n_{\nu}+n_{\mu} k_{\nu}}{n k}
$$

for the virtual gluon, we make the total contribution of all three graphs in Fig.2 to be concentrated in the single graph (a). Iterating this graph, we obtain all graphs (of the ladder type only) contributing to $f_{N S}$. In order to avoid the infrared (IR) singularities that appear in the radiative corrections, we use Lipatov's prescription of compactifying the impact parameter space (see e.g. [7] 9]), in other words, we introduce the infrared cut-off $\mu$ in the transverse space (with respect to the plane formed by $q$ and $p$ ) when integrating over the momenta of virtual particles. With such a cut-off acting as a mass scale, one can neglect quark masses and still be free from IR singularities. Until now the procedure is not different from the one used in the DGLAP, apart from the infrared regularization, which is a purely technical issue. The main difference comes when specifying the limits of integration over the transverse momenta of virtual particles. In the DGLAP, all ladder transverse momenta $k_{i \perp}$ are ordered as

$$
k_{1 \perp} \leq k_{2 \perp} \leq \ldots \leq Q^{2},
$$


with the numeration running from the bottom of the ladder to the top.

Equation (4) shows that only the integration over $k_{1 \perp}$ has $\mu$ as the lower limit. The lower limits for other $k_{i \perp}$ with $i \neq 1$ are expressed through the transverse momenta. As is well known, the ordering of Eq. (4) leads to considering the logarithms of $Q^{2}$ only. On the other hand, as we investigate the small- $x$ region, we will lift this ordering, allowing for the transverse momentum of quarks in any ladder rung to reach the lowest limit $k_{\perp}=\mu$. Obviously, this increases the region of integration over $k_{i \perp}$. Let us represent any graph contributing to $M$ as a convolution of two amplitudes connected by the rung with minimal $k_{\perp}$. In the DGLAP, such a representation is given by the graph in Fig.3, with the minimal $k_{\perp}$ in the lowest rung only; now, having lifted the $k_{\perp}$ ordering of Eq. (4), in the same graph, the minimal $k_{\perp}$ is assigned to any rung. Summing up such graphs to all loops and adding the Born graph, one arrives at the equation represented in Fig. Ia if we use the DGLAP ordering and at that of Fig. 四 if one drops the $k_{\perp}$ ordering. The blobs in Fig. 1 denote that radiative corrections to all orders in QCD coupling are taken into account. As a result of the infrared regularization we have chosen, the amplitude $M$ is $\mu$-dependent now and one can evolute $M$ with respect to $\mu$. It is convenient to use the Mellin transform for the amplitude $M:$

$$
M\left(s / \mu^{2}, Q^{2} / \mu^{2}\right)=\int_{-\imath \infty}^{\imath \infty} \frac{d \omega}{2 \pi \imath}\left(\frac{s}{\mu^{2}}\right)^{\omega} F\left(\omega, Q^{2} / \mu^{2}\right) .
$$

As we are going to take into account both logarithms of $s / \mu^{2}$ and of $Q^{2} / \mu^{2}$ in every order in $\alpha_{s}$, we assume that

$$
M=M^{\text {Born }} U\left(\ln \left(s / \mu^{2}\right), \ln \left(Q^{2} / \mu^{2}\right)\right),
$$

where

$$
M^{\text {Born }}=-\frac{s}{s-Q^{2}+\imath \epsilon}
$$

and $U$ has to be properly defined. Equation (7) leads to $\Im_{s} M^{B o r n}=\pi \delta(1-x)$, which agrees with Eqs. (1) and (21). 
Let us note that $U$ must have a non-zero imaginary part, $\Im_{s} U$, when $s$ is positive and $\Im_{s} U=0$ when $s$ is negative. This is satisfied when

$$
U=U\left(\ln \left(-s / \mu^{2}\right), \ln \left(Q^{2} / \mu^{2}\right)\right)=U\left(\left(\ln \left(s / \mu^{2}\right)-\imath \pi\right), \ln \left(Q^{2} / \mu^{2}\right)\right)
$$

Since $\ln \left(s / \mu^{2}\right)$ is assumed to be much greater than $\pi$, one can expand Eq. (8) in the small- $x$ limit as follows:

$$
M=(-1+\imath \pi \delta(1-x))\left[U\left(\ln \left(s / \mu^{2}\right), \ln \left(Q^{2} / \mu^{2}\right)\right)-\imath \pi \frac{\partial U\left(\ln \left(s / \mu^{2}\right), \ln \left(Q^{2} / \mu^{2}\right)\right)}{\partial \ln \left(s / \mu^{2}\right)}+\ldots\right]
$$

so that

$$
\Im_{s} M \approx \pi\left[\delta(1-x) U\left(\ln \left(Q^{2} / \mu^{2}\right)\right)+\frac{\partial U}{\partial \ln \left(s / \mu^{2}\right)}\right] .
$$

Combining (10) and (5), we obtain, in the small- $x$ limit,

$$
\left.f_{N S}=\int_{-\imath \infty}^{\imath \infty} \frac{d \omega}{2 \pi \imath}\left(\frac{s}{\mu^{2}}\right)^{\omega} \omega F_{(} \omega, Q^{2} / \mu^{2}\right)
$$

So, once we know $F$ we also know $f_{N S}$. Now we calculate $F$ using the equations in Fig. 4 . Differentiating the left-hand sides of the equations in Fig. that

$$
-\mu^{2} \frac{\partial M}{\partial \mu^{2}}=\frac{\partial M}{\partial \ln \left(s / \mu^{2}\right)}+\frac{\partial M}{\partial \ln \left(Q^{2} / \mu^{2}\right)}
$$

corresponds to

$$
\omega F+\frac{\partial F}{\partial y}
$$

for the Mellin amplitude $F$. We have defined $y=\ln \left(Q^{2} / \mu^{2}\right)$.

Then, differentiating with respect to $\ln \left(\mu^{2}\right)$ the right-hand sides of the two equations in Fig. 4 , we arrive at the equation (the Born contribution does not depend on $\mu$ and cancels under the differentiation):

$$
\left(\frac{\partial}{\partial y}+\omega\right) \widetilde{F}(\omega, y)=\left[\frac{1}{8 \pi^{2}}(1+\lambda \omega)\right] \widetilde{F}(\omega, y) \widetilde{F}_{0}^{B}(\omega)
$$


if we follow the DGLAP $k_{\perp}$ ordering (4), and at the equation

$$
\left(\frac{\partial}{\partial y}+\omega\right) F(\omega, y)=\left[\frac{1}{8 \pi^{2}}(1+\lambda \omega)\right] F(\omega, y) F_{0}(\omega)
$$

if we do not invoke this $k_{\perp}$ ordering.

In Eqs. (14) and (15) we have used the notations $\lambda=1 / 2$ and provided the DGLAP amplitudes with a tilde. The expression in the squared brackets is the result of a one-loop integration in the right-hand side of the equations represented in Fig.4.

The Born DGLAP amplitude for the forward scattering of quarks

$$
\widetilde{F}_{0}^{B}=4 \pi \alpha_{s}\left(Q^{2}\right) C_{F} / \omega
$$

with $C_{F}=\left(N^{2}-1\right) / 2 N=4 / 3$, whereas the amplitude $F_{0}(\omega)$ for the forward scattering of quarks in Eq. (15) is unknown yet and has to be calculated independently.

Equation (14) leads to the DGLAP result for the non-singlet structure function in the leading order. Indeed, the solution of Eq. (14) is

$$
\widetilde{F}=\widetilde{C} \exp \left(\int_{\mu^{2}}^{Q^{2}} \frac{d r}{r} \frac{\alpha_{s}(r) C_{F}}{2 \pi}\left[\frac{1}{\omega}+\lambda\right]\right),
$$

and we therefore arrive at the famous leading-order DGLAP formula for $f_{N S}$ :

$$
\tilde{f}_{N S}=\int_{-\imath \infty}^{\imath \infty} \frac{d \omega}{2 \pi \imath}\left(\frac{1}{x}\right)^{\omega} \widetilde{C} \exp \left(\widetilde{A}\left[\frac{1}{\omega}+\lambda\right]\right)
$$

with $\widetilde{A}$ given by

$$
\widetilde{A}=\frac{C_{F}}{2 \pi} \int_{\mu^{2}}^{Q^{2}} \frac{d r}{r} \alpha_{s}(r)
$$

The quantity $\widetilde{C}$ is not determined a priori and has to be fixed somehow. However, if one assumes the delta-function input (1) corresponding to DIS off a quark in the Born approximation, one can specify $\widetilde{C}$ by the matching:

$$
\widetilde{M}=\widetilde{M^{B}}
$$

when $y=0$, i.e. 


$$
\widetilde{C}=1
$$

Then one can finalize the leading-order DGLAP result for the non-singlet structure function with the delta-function input (24) as:

$$
\tilde{f}_{N S}=\int_{-\imath \infty}^{\imath \infty} \frac{d \omega}{2 \pi \imath} \exp \left(\tilde{A}\left[\frac{1}{\omega}+\lambda\right]\right) .
$$

On the other hand, in order to consider the small- $x$ behaviour of $f_{N S}$, we have to lift the $k_{\perp}$ ordering of Eq. (4) and to use Eq. (15) rather than Eq. (14). The solution to (15) is

$$
F=C \exp \left(\left[-\omega+(1+\lambda \omega) F_{0}(\omega) / 8 \pi^{2}\right] y\right)
$$

which contains two unknown quantities, $C$ and $F_{0}$, which have to be specified.

Just as for the DGLAP, input $C$ can be fixed by making special assumptions. Then the expression for $f_{N S}$ at small $x$ is given by the Mellin transform of Eq. (11), with the integrand Eq. (23), provided $F_{0}$ is known. In the case of the delta-function input, one can specify $C$. Equation (23) shows that $C$ actually coincides with the Mellin amplitude $M$ when the incoming photon is almost on-shell, i.e. its virtuality is $\mu^{2}$. So the equation for $C$ is similar to the one for $M$, but it differs from (15) on two points: first, there is no $Q^{2}$-dependence in the left-hand side; second, the Born contribution to $C$ is $\mu$-dependent. We recall that the Born contribution to $M$ was independent of $\mu$ (see (11)) and therefore it vanished under differentiation over $\mu$. Now we have to add the Born contribution

$$
1 / \omega
$$

to the right-hand side of the equation for $C$. Thus, $C$ obeys

$$
C(\omega)=\frac{1}{\omega}+\frac{1}{8 \pi^{2}}[1 / \omega+\lambda] C(\omega) F_{0}(\omega)
$$

with the obvious solution

$$
C=1 /\left[\omega-(1+\lambda \omega) F_{0}(\omega) / 8 \pi^{2}\right]
$$

So, the only ingredient that still remains unknown in the right-hand side of Eq. (23) is the amplitude $F_{0}$, corresponding to the scattering of quarks. 
The equation for $F_{0}$ is shown in Fig.5. It looks pretty similar to Eq. (25) for $C$. The difference is in the Born amplitude $A(\omega) / \omega$ for the quark scattering, which we specify below in Eq. (40). Then, in analogy to Eq.(25), we obtain

$$
F_{0}(\omega)=\frac{A(\omega)}{\omega}+\frac{1}{8 \pi^{2}}\left[\frac{1}{\omega}+\lambda\right] F_{0}^{2}(\omega)
$$

which has the solution

$$
F_{0}=4 \pi^{2} \frac{\left[\omega-\sqrt{\omega^{2}-(1+\lambda \omega) A(\omega) / 2 \pi^{2}}\right]}{1+\lambda \omega} .
$$

Finally, combining the above results, we obtain the expression for the non-singlet structure function (cf. (17)):

$$
f_{N S}=\int_{-\imath \infty}^{\imath \infty} \frac{d \omega}{2 \pi \imath} C\left(\frac{1}{x}\right)^{\omega} \omega \exp \left(\left[(1+\lambda \omega) F_{0} / 8 \pi^{2}\right] y\right)
$$

for an arbitrary input $C$, or

$$
f_{N S}=\int_{-\imath \infty}^{\imath \infty} \frac{d \omega}{2 \pi \imath}\left(\frac{1}{x}\right)^{\omega} \omega \frac{F_{0}(\omega)}{A(\omega)} \exp \left(\left[(1+\lambda \omega) F_{0} / 8 \pi^{2}\right] y\right)
$$

where we have used Eqs. (26) and (28) and $A(\omega)$ is unspecified yet. Now let us determine A. In doing so we use the approach suggested in [8], with some important changes.

Let us consider the forward quark-scattering amplitude in the Born approximation. The only Feynman graph with a non-zero discontinuity (imaginary part) with respect to the Mandelstam total energy variable $s^{\prime}$ of this process is shown in Fig.6. It yields

$$
M_{0}^{B}=-4 \pi \alpha_{s} C_{F} \frac{s^{\prime}}{s^{\prime}+\imath \epsilon}
$$

with $\alpha_{s}$ fixed in the Born approximation.

Among the radiative corrections to the Born amplitude, there are those, see Fig.7, that make $\alpha_{s}$ running. The contributions of the graphs of that kind lead to replacing $M_{0}^{B}$ by $B$, when the QCD coupling depends on $s^{\prime}$ :

$$
B=-4 \pi \alpha_{s}\left(s^{\prime}\right) C_{F} \frac{s^{\prime}}{s^{\prime}+\imath \epsilon}
$$


Furthermore, in the planar gauge, taking into account the radiative corrections is equivalent to iterating $B$ (we recall that we discuss the positive signature in the present paper). As a result of such an iteration we arrive at the amplitude $M_{0}\left(s^{\prime}\right)$. In order to avoid the IR singularities in $M_{0}$, we again use the IR cut-off $\mu$ so that $s^{\prime} \geq \mu^{2}$. in the denominators of Eqs. (31) and (32) Equivalently, it can be expressed by replacing $s^{\prime}+\imath \epsilon$ by $s^{\prime}-\mu^{2}+\imath \epsilon$ in the denominators in (31) and (32). One can now apply the Mellin transform to $B$ :

$$
B=\int_{-\imath \infty}^{\imath \infty} \frac{d \omega}{2 \pi \imath}\left(\frac{s}{\mu^{2}}\right)^{\omega} R(\omega)
$$

In order to invert $R(\omega)$ through $B(s)$, one must use the inverse transform in Eq. (33). While the Mellin transform (5) is the extreme high-energy limit of the Sommerfeld - Watson transform, the inverse transform, which respects the analytical properties of the scattering amplitude under discussion, involves its discontinuities (see e.g. [14]). Then, it is appropriate to use this inverse transform in a form similar to the one suggested in [8]. The inverse transform of Eq. (5) is

$$
F(\omega)=-\frac{1}{\sin (\pi \omega)} \int_{0}^{\infty} d \rho \exp (-\omega \rho) \Im_{s} M(\rho)
$$

where $\rho=\ln \left(s / \mu^{2}\right)$, we will approximate $\sin (\pi \omega) \approx \pi \omega$ at small $\omega$. Then inverting Eq. (33) we obtain

$$
R(\omega)=-\frac{1}{\pi \omega} \int_{0}^{\infty} d \rho e^{-\omega \rho} \Im_{s} B(\rho)
$$

having defined $\rho=\ln \left(s / \mu^{2}\right)$.

Using the standard one-loop form for $\alpha_{s}$

$$
\alpha_{s}(s)=\frac{1}{b \ln \left(-s / \Lambda_{Q C D}^{2}\right)},
$$

when $s>0$, we obtain

$$
R(\omega)=\frac{4 C_{F} \pi}{b \omega} \int_{0}^{\infty} d \rho\left[\frac{1}{(\rho+m)^{2}+\pi^{2}}-\frac{(\rho+m)}{\left[(\rho+m)^{2}+\pi^{2}\right]}\left(\frac{s}{\mu^{2}}\right) \delta\left(s / \mu^{2}-1\right)\right] \exp (-\rho \omega),
$$


where

$$
m=\ln \left(\mu^{2} / \Lambda_{Q C D}^{2}\right) ;
$$

and we assume $\mu>\Lambda_{Q C D}$. The first term in the square brackets in Eq. (37) corresponds to the imaginary part of (36) and the second one comes from the imaginary part of $1 /\left(s-\mu^{2}+\imath \epsilon\right)$.

We rewrite the expression for $R$ in terms of $A$ that we introduced in (27):

$$
R(\omega)=\frac{A}{\omega}
$$

with

$$
A(\omega)=\frac{4 C_{F} \pi}{b}\left[\frac{m}{m^{2}+\pi^{2}}-\int_{0}^{\infty} \frac{d \rho \exp (-\rho \omega)}{(\rho+m)^{2}+\pi^{2}}\right] .
$$

Comparing Eq. (40) with the inverse Mellin transform of Eq. (31), we see that $A(\omega)$ corresponds to running $\alpha_{s}$ in the Mellin space. In the next section we will discuss Eq. (40) in some more detail.

\section{ASYMPTOTICS OF $F_{N S}$}

Before making use of all results obtained in the previous section, let us dwell upon our expression for $R$ and $A$. Equations (39) and (40) differ from the similar expressions in [8] on one essential point: $\pi^{2}$ was not accounted for in either [8] or [\$, 6], though its numerical value is quite large with respect to other terms in the integrand and cannot be neglected a priori.

As stated above, $A$ in (40) stands for the QCD coupling in the Mellin space. Indeed, if we let $\alpha_{s}$ be fixed just as in the DLA, we obtain

$$
R_{D L}=\frac{4 \pi C_{F} \alpha_{s}^{D L}}{\omega}
$$

instead of (40). We note that in the framework of DGLAP, we would have obtained $\widetilde{A}$ given by (19) instead of $A / 8 \pi^{2}$ just as it was assumed in [10 [12]. The reason of such a discrepancy is obvious: in the ladder Feynman graphs, the current argument for $\alpha_{s}$ in the $n$-th ladder 
rung is, actually, $\beta_{n+1} k_{n \perp}^{2} / \beta_{n}$ t. Only when $x \sim 1$ do we have $\beta_{n+1} \sim \beta_{n}$, and therefore all $\beta$ can be dropped in the arguments of $\alpha_{s}$ in every rung. Then, the DGLAP ordering (田) establishes $Q^{2}$ as the upper limit of integration over $k_{\perp}^{2}$. With the above approximations, one arrives in due course at (19). However, neither of those approximations holds in the small- $x$ region, where there is no ordering in $k_{\perp}$ and the upper limit is $s$ rather than $Q^{2}$. Dropping those approximations leads to the expression for $f_{N S}$ at small $x$ given by the expressions in Eqs. (29) and (40) for an arbitrary input and by Eqs. (30) and (40) when the delta-function input is used.

It is also easy to check that the corresponding DGLAP expressions can be obtained from Eqs. (29) and (30). Indeed, when $x \sim 1$, the main contribution of the Mellin factor, $\exp [\omega \ln (1 / x)]$, comes from the region of rather large values of $\omega$, where $\omega \ln (1 / x) \leq 1$. That makes it possible to expand the exponent $F_{0}$ in Eq. (29) into a series in $1 / \omega$. Doing so, we obtain

$$
(1+\lambda \omega) F_{0} / 8 \pi^{2}=\frac{A}{8 \pi^{2}}\left[\frac{1}{\omega}+\lambda\right]+\left(\frac{A}{8 \pi^{2}}\right)^{2} \frac{1}{\omega}\left[\frac{1}{\omega}+\lambda\right]^{2}+O\left(A^{3}\right) .
$$

Retaining only the first term, proportional to $A$ in the right-hand side of Eq. (42), and combining the result with Eq. (29) one arrives at the expression for $f_{N S}$, which is similar to the DGLAP expression in Eq. (18). In order to get a complete coincidence, $y A / 8 \pi^{2}$ has to be replaced by $\widetilde{A}$ as given by Eq. (18). The same expansion of the integrand into a 1/ $\omega$-series relates Eq. (30) and Eq. (22) for $f_{N S}$ with the delta-function input (1).

The small- $x$ asymptotic behaviour of $f_{N S}$ can be obtained by evaluating $f_{N S}$ with the saddle-point method. As the asymptotics of the integrals (29) and (30) are identical, we consider below expression (29), which we rewrite as follows:

\footnotetext{
${ }^{1} k_{n \perp}$ and $\beta$ are the standard Sudakov variables for ladder virtual quark momenta. For the Sudakov parametrization in the context of $f_{N S}$, see e.g. [4,5].
} 


$$
f_{N S}=C \int_{-\imath \infty}^{\infty \infty} \frac{d \omega}{2 \pi \imath} \exp \left[\omega \xi+\frac{y}{2}\left(\omega-\sqrt{\omega^{2}-(1+\lambda \omega) A(\omega) / 2 \pi^{2}}\right)\right],
$$

where we have used the notation $\xi=\ln (1 / x)$ and $y=\ln \left(Q^{2} / \mu^{2}\right)$. According to the saddlepoint method, when $x$ tends to zero,

$$
f_{N S} \sim f_{N S}\left(\omega_{0}\right)
$$

where the stationary point $\omega_{0}$ is the largest of the roots of

$$
\frac{d}{d \omega}\left[\omega \xi+\frac{y}{2}\left(\omega-\sqrt{\omega^{2}-\left(1+\lambda \omega A / 2 \pi^{2}\right)}\right)\right]=0 .
$$

Differentiating in (45) leads to

$$
\left[\omega^{2}-(1+\lambda \omega) A / 2 \pi^{2}\right]\left(\frac{2 \xi}{y}+1\right)^{2}=\left[\omega-\lambda A / 4 \pi^{2}-(1+\lambda \omega)(d A / d \omega) /\left(4 \pi^{2}\right)\right]^{2},
$$

which can be solved numerically for any $\xi, y$. However, for obtaining asymptotically small- $x$ behaviour a further simplification is possible: when $\xi / y \gg 1$, the right-hand side of Eq. (46) is small with respect to the left-hand side and therefore $\omega_{0} \approx \omega_{s}$, where $\omega_{s}$ is the root of

$$
\omega^{2}-(1+\lambda \omega)\left(A / 2 \pi^{2}\right)=0 .
$$

In other words, $\omega_{0}$ is the position of the leading singularity (the branch point) of the exponent in Eq. (43) in the $\omega$ plane. So, with the square root in (43) being zero when $\omega=\omega_{0}$, we arrive at the power-like asymptotics:

$$
f_{N S} \sim f_{N S}\left(\omega_{0}\right)=\exp \left[\omega_{0} \xi+y \omega_{0} / 2\right]=\left(\frac{1}{x}\right)^{\omega_{0}}\left(\frac{Q^{2}}{\mu^{2}}\right)^{\omega_{0} / 2} .
$$

On the other hand, if one expands the exponent in (43) into a series in $1 / \omega$ (see (42)) and accounts for an arbitrary but finite number of terms in the series, one arrives at the well-known DGLAP asymptotics for $f_{N S}$ instead of the power-like one. For example, let us retain only the first term in (42). In this case $\omega_{0}$ is the root of

$$
\frac{d}{d \omega}\left[\omega \xi+y\left(A / 8 \pi^{2}\right)\left(\frac{1}{\omega}+\lambda\right)\right]=0 .
$$


Replacing $\left(y A / 8 \pi^{2}\right)$ by $\tilde{A}\left(Q^{2}\right)$ (cf. (18)) in order to treat the QCD coupling as in the DGLAP, we obtain that the leading singularity $\tilde{\omega}_{0}$ is now

$$
\tilde{\omega}_{0}=\sqrt{\tilde{A} / \xi}
$$

therefore, in this approximation :

$$
f_{N S} \sim f_{N S}\left(\omega_{0}\right)=e^{\sqrt{\tilde{A} \ln (1 / x)}}
$$

Thus, taking into account a finite number of NLO terms in the expressions for the anomalous dimensions inevitably leads to the asymptotic behaviour of the DGLAP type, whereas the power-like behaviour is the result of accounting for the NLO contributions to all orders in $\alpha_{s}$. Now let us calculate the exponent in Eq. (48). Using Eq. (40), we eventually obtain the equation for the position of the leading singularity $\omega_{s}$ when $x$ tends to zero :

$$
\omega^{2}-(1+\lambda \omega)\left(\frac{2 C_{F} \pi}{b}\right)\left[\frac{m}{m^{2}+\pi^{2}}-\int_{0}^{\infty} \frac{d \eta e^{-\omega \eta}}{(\eta+m)^{2}+\pi^{2}}\right]=0 .
$$

Here, $b$ is the first coefficient of the $\beta$-function $: b=\left(33-2 n_{f}\right) / 12 \pi$. We recall that $m$ in (52) stands for $\ln \left(\mu^{2} / \Lambda_{Q C D}^{2}\right)$ and that we keep the relation $\mu>\Lambda_{Q C D}$ throughout this paper.

It is easy to solve Eq. (52) numerically, for any fixed set of parameters $n_{f}, \mu, \Lambda_{Q C D}$. For example, using $n_{f}=3, \mu=1 \mathrm{GeV}, \Lambda_{Q C D}=0.1 \mathrm{GeV}$, we obtain

$$
\omega_{s}=0.37
$$

On the other hand, the small- $x$ behaviour of the non-singlet structure functions could be calculated in the double-logarithmic approximation and is given by

$$
f_{N S} \sim x^{-\omega_{D L}}
$$

with the exponent

$$
\omega_{D L}=\sqrt{2 \alpha_{s}^{D L} C_{F} / \pi}
$$


(see [4 for details). The QCD coupling $\alpha_{s}^{D L}$ is of course fixed in the DLA and its value cannot be specified within the DLA accuracy. Nevertheless, as asymptotically the DLA is supposed to dominate eventually over subleading contributions, we can estimate such an "asymptotic value" $\alpha_{s}^{D L}$ by identifying $\omega_{s}$ and $\omega_{D L}$. Doing so, we obtain

$$
\alpha_{s}^{D L}=\omega_{s}^{2} \pi / 2 C_{F}
$$

In particular, for the same set of parameters as given above, we obtain $\alpha_{s}^{D L}=0.16$ which is the value of $\alpha_{s}\left(60 \mathrm{GeV}^{2}\right)$. We note that this value is not at all related to the value of $\alpha_{s}\left(Q^{2}\right)\left(Q^{2}\right.$ being the incoming photon virtuality), which has often been used in the DLA calculations as an estimate for the value of $\alpha_{s}^{D L}$. We would like to further comment on the dependence of $\omega_{s}$ on the approximation used in our calculation. First, if we had neglected the single-logarithmic contributions, putting $\lambda=0$ and also dropping the $\pi^{2}$ terms in Eq. (52), we would have obtained $\omega_{s}=0.43$. It corresponds to $\alpha_{s}^{D L}=0.22=\alpha_{s}\left(6 G e v^{2}\right)$. On the other hand, keeping both double- and single-logarithmic contributions (i.e. $\lambda=1 / 2$ ) and neglecting the $\pi^{2}$ terms, we would get $\omega_{s}=0.49$ and therefore, for this case, $\alpha_{s}^{D L}=0.28=$ $\alpha_{s}\left(1.5 \mathrm{Gev}^{2}\right)$. It is clear that the $\pi^{2}$ terms, which come from analyticity, account only for a class of constant terms and should in principle, be neglected for consistency. However, the importance of keeping them into account in resummation formulae has been stressed for a long time [16]. Unfortunately we have not been able to compare the higher terms in the expansion of $f_{N S}$ Eq. (42) with fixed higher orders in $\alpha_{s}$ calculations since the subleading (the single-logarithmic) contributions to $f_{N S}$, even in the first loop depend on the choice of the factorization and the regularization procedures. It would be very interesting to see the effect of SL contributions to the $f_{N S}$ with the negative signature (these contributions appear as from the order $\alpha_{s}^{3}$ ). At present, such calculations are still in progress. Although we have called Eqs. (29) and (30) a generalization of the leading-order DGLAP, those expressions actually include also the singular contributions in $\omega$ present in the expressions for the twoloop DGLAP anomalous dimension for $f_{N S}$ as well as other singular terms coming from the resummation of the leading-order DGLAP anomalous dimension to all orders in $\alpha_{s}$. 


\section{CONCLUSIONS}

In conclusion, we have obtained a generalization of the leading-order DGLAP for $f_{N S}$ to the case of small $x$, by resumming the leading-order DGLAP anomalous dimension $1 / \omega+1 / 2$ to all orders in $\alpha_{s}$ and by accounting for running $\alpha_{s}$ effects. In addition to the doublelogarithmic contributions we took into account the single-logarithmic ones. Our result for the first-loop contribution to $f_{N S}$ is in agreement with [15], provided that identical regularizations are used. We have demonstrated above that our results of Eqs. (29) for an arbitrary input and (30) for the delta-function input (1) agree with the DGLAP when we neglect all terms except the first one in Eq. (42). We showed that, with the single logarithmic contributions and with running $\alpha_{s}$ taken into account, $f_{N S}$ also has the power-like asymptotic behaviour

$$
f_{N S} \sim x^{-\omega_{s}}\left(Q^{2} / \mu^{2}\right)^{\omega_{s} / 2}
$$

as was obtained earlier in the DLA [4] at asymptotically small $x$ but with another value of the exponent. We have also investigated the dependence of the value of $\omega_{s}$ on the accuracy of the calculations. In doing so we obtained that subleading (single-logarithmic) terms contribute to the asymptotic behaviour of $f_{N S}$ by increasing $\omega_{s}$ in Eq. (57) from its value $\omega_{s}=0.43$ in the DLA up to $\omega_{s}=0.49$. On the contrary, the $\pi^{2}$-terms strongly suppress that growth, decreasing the value of $\omega_{s}$ down to $\omega_{s}=0.37$. This result can be regarded as an indication for the importance of the subleading contributions. In the present work we have discussed mainly the asymptotic behaviour of $f_{N S}$. A detailed comparison of our results with the DGLAP at small but finite values of $x$ will be given elsewhere.

\section{ACKNOWLEDGEMENTS}

We are grateful to G. Altarelli, S. Catani and S. Forte for useful discussions. This work suppoorted in part by the EU QCDNET contract FMRX-CT98-0194. 


\section{REFERENCES}

[1] G.Altarelli and G.Parisi, Nucl. Phys.B126 (1977) 297; V.N.Gribov and L.N.Lipatov, Sov. J. Nucl. Phys. 15 (1972) 438; L.N.Lipatov, Sov. J. Nucl. Phys. 20 (1972) 95; Yu.L.Dokshitzer, Sov. Phys. JETP 46 (1977) 641.

[2] G.Altarelli, R.D.Ball, S.Forte and G.Ridolfi, Nucl. Phys. B496 (1997) 337.

[3] V.S.Fadin, E.A.Kuraev and L.N.Lipatov, Sov. Phys. JETP 44 (1976) 443; and 45 (1977) 199; Y.Y.Balitskij and L.N.Lipatov, Sov. J. Nucl. Phys. 28 (1978) 822.

[4] B.I.Ermolaev, S.I.Manayenkov and M.G.Ryskin, Z. Phys. C69 (1996) 259.

[5] J.Bartels, B.I.Ermolaev and M.G.Ryskin, Z. Phys.C70 (1996) 273 and C72 (1996) 627.

[6] S.I.Manayenkov, Z. Phys. C75 (1997) 685.

[7] L.N.Lipatov, Phys. Lett. B116 (1982) 411.

[8] R.Kirschner and L.N.Lipatov, Nucl. Phys. B213 (1983) 122.

[9] B.I.Ermolaev and L.N.Lipatov, Int. J. Mod. Phys. A4 (1989) 3147.

[10] B.Badalek and J.Kwiecinski, Phys. Lett. B418 (1998) 229.

[11] J.Kwiecinski and B.Ziaja, hep-ph/9902440.

[12] J.Blumlein, S.Riemersma and A.Vogt, Acta Phys. Pol. B28 (1997) 577; J.Blumlein and A.Vogt, Acta Phys. Pol. B27 (1996) 1309; J.Blumlein and A.Vogt, Phys. Lett. B370 (1996) 149 .

[13] Y.Kiyo and J.Kodaira, hep-ph/9803448; hep-ph/9711260; Z. Phys. C74 (1997) 631.

[14] P.D.B.Collins, An introduction to Regge theory and high energy physics. Cambridge, 1977.

[15] G.Curci, W.Furmanski and R.Petronzio, Nucl. Phys. B175 (1980) 27. 
[16] G.Curci and M.Greco, Phys. Lett. 92B (1980) 175.

\section{Figure captions}

Fig.1 The Born graphs for the amplitude $M$.

Fig.2 The graphs contributing to $f_{N S}$ in one-loop approximations.

Fig.3 A typical graph for $f_{N S}$ in the planar gauge.

Fig.4 The evolution equation for $M$.

Fig.5 The evolution equation for the quark scattering amplitude.

Fig.6 The Born graph for the quark scattering amplitude.

Fig.7 The graphs contributing to the running QCD coupling. 


\section{FIGURES}

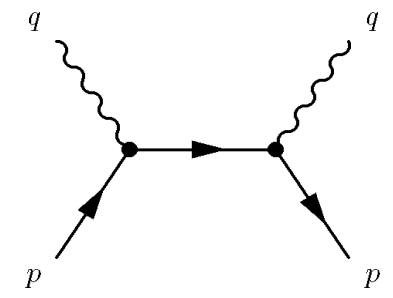

(a)

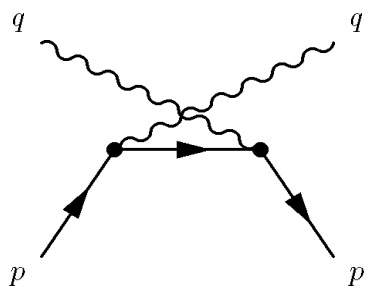

(b)

FIG. 1. The Born graphs for the amplitude $M$.

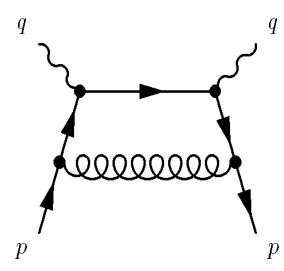

(a)

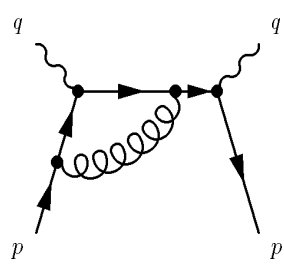

(b)

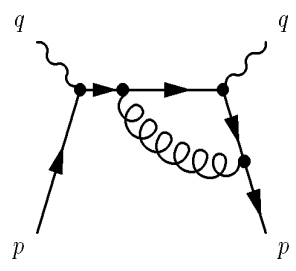

(c)

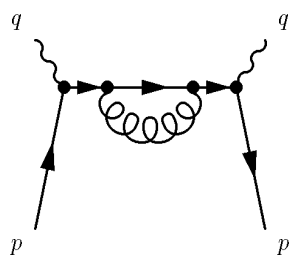

(d)

FIG. 2. The graphs contributing to $f_{N S}$ in one-loop approximations.

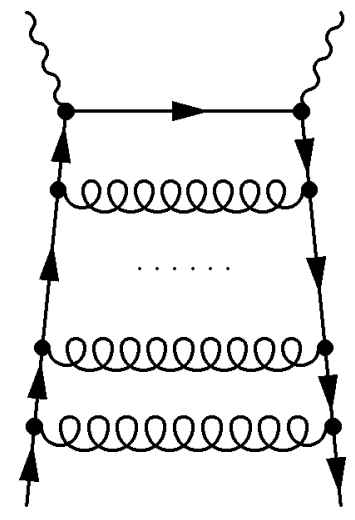

FIG. 3. A typical graph for $f_{N S}$ in the planar gauge. 


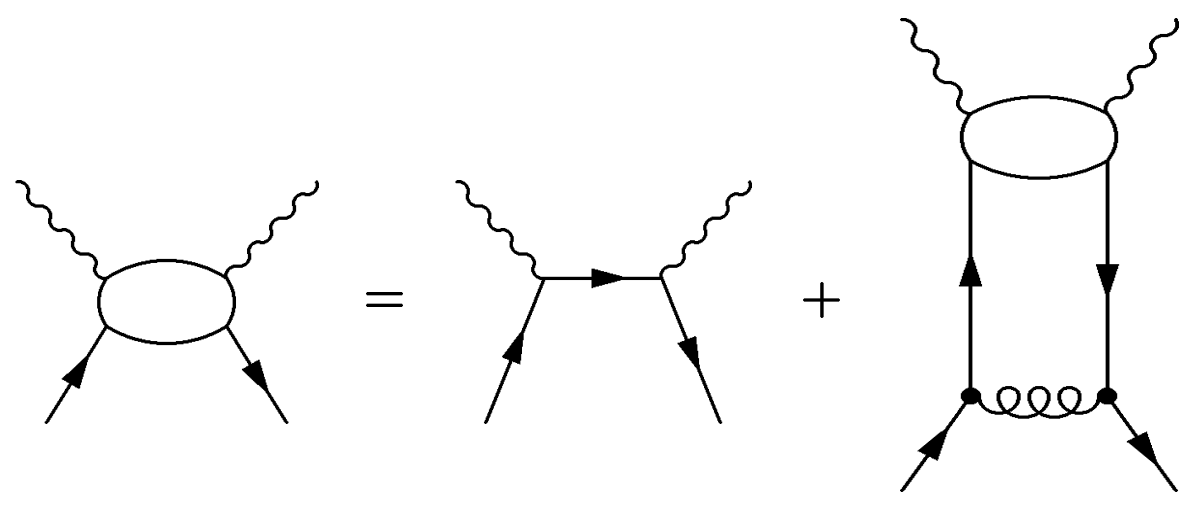

(a)
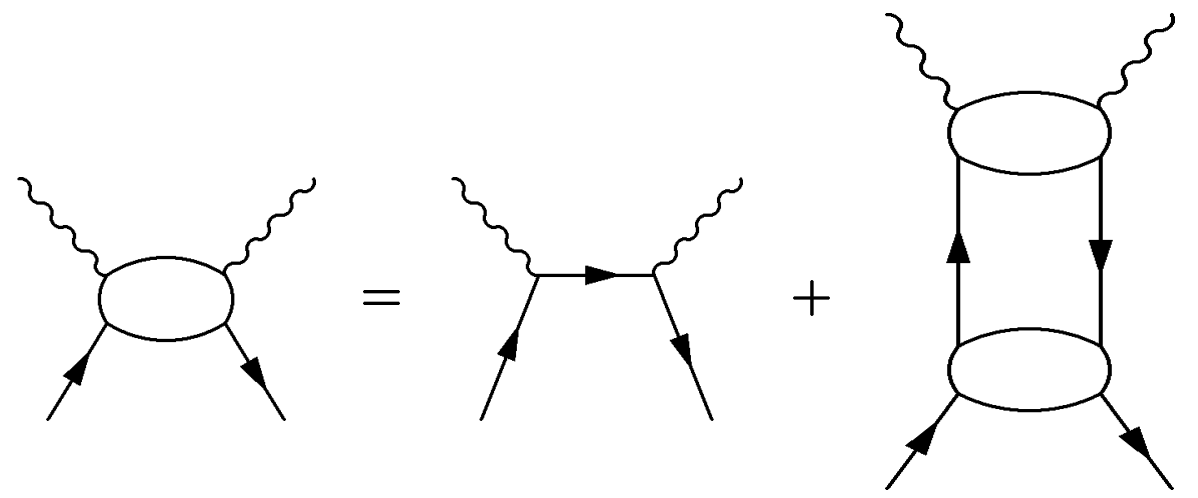

(b)

FIG. 4. The evolution equation for $M$.

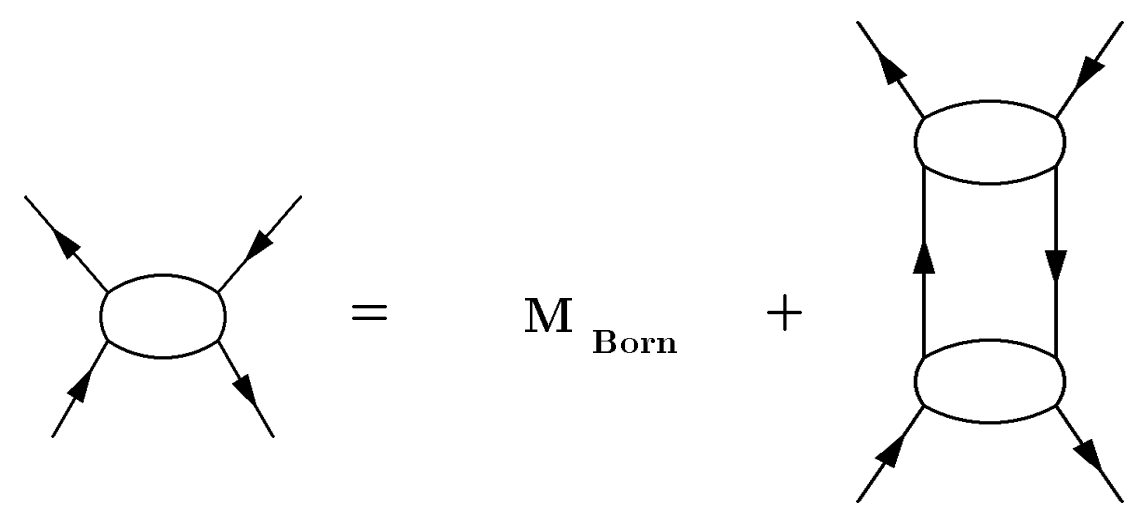

FIG. 5. The evolution equation for the quark scattering amplitude. 


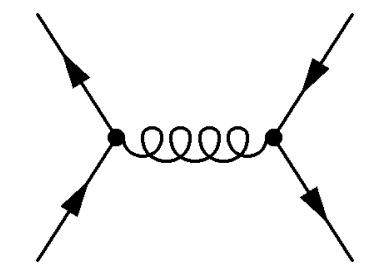

FIG. 6. The Born graph for the quark scattering amplitude.
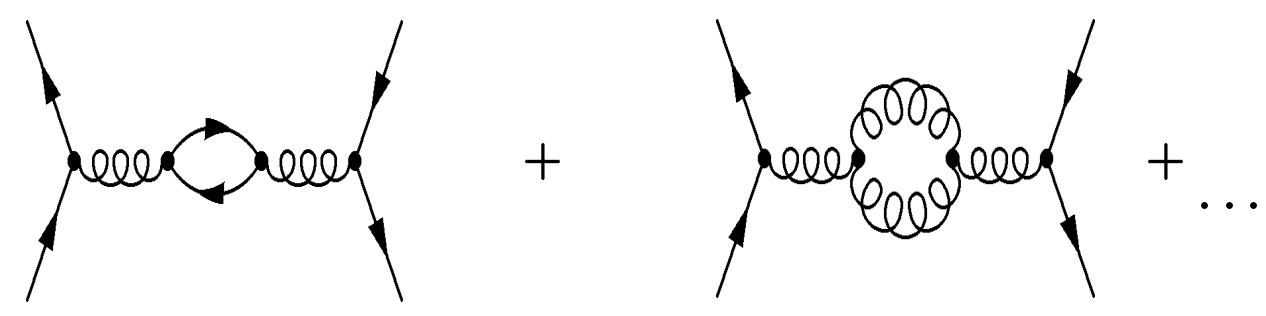

FIG. 7. The graphs contributing to the running QCD coupling. 